\title{
Attractor Flows from Defect Lines
}

\author{
Ilka Brunner ${ }^{1,2 *}$ and Daniel Roggenkamp ${ }^{3 \dagger}$ \\ ${ }^{1}$ Arnold Sommerfeld Center, Ludwig Maximilians Universität \\ Theresienstr. 37, 80333 München, Germany \\ ${ }^{2}$ Excellence Cluster Universe, Technische Universität München \\ Boltzmannstr. 2, 85748 Garching, Germany \\ ${ }^{3}$ Department of Physics and Astronomy, Rutgers University \\ Piscataway, NJ 08855-0849, USA
}

\begin{abstract}
Deforming a two dimensional conformal field theory on one side of a trivial defect line gives rise to a defect separating the original theory from its deformation. The Casimir force between these defects and other defect lines or boundaries is used to construct flows on bulk moduli spaces of CFTs. It turns out, that these flows are constant reparametrizations of gradient flows of the $g$-functions of the chosen defect or boundary condition. The special flows associated to supersymmetric boundary conditions in $N=(2,2)$ superconformal field theories agree with the attractor flows studied in the context of black holes in $N=2$ supergravity.
\end{abstract}

\footnotetext{
*E-mail: Ilka.Brunner@physik.uni-muenchen.de
}

${ }^{\dagger}$ E-mail: roggenka@physics.rutgers.edu 


\section{Contents}

1. Introduction 1

2. Deformation defects and flows on bulk moduli spaces 3

3. Example: The free boson 10

4. Supersymmetric version and attractor flows 21

A. Perturbative variation of charges 23

B. The Hessian of $\ln \left(g_{\mathcal{D}}\right)$ for topological defects 24

C. Vanishing of bulk-boundary/-defect couplings for $N=2$ theories

\section{Introduction}

Conformal defects are lines of inhomogeneity between two possibly different two dimensional conformal field theories which preserve conformal invariance. In this article, defect lines are used to associate flows on moduli spaces of conformal field theories to given boundary conditions or defects in these theories. They arise in the following way. As has been discussed in the context of Landau-Ginzburg models in $[1,2]$, to each bulk perturbation of a conformal field theory one can associate a unique defect line between the IR and the UV theories of the corresponding RG flow. It is obtained by performing the perturbation on one side of the trivial identity defect of the UV theory. Applied to exactly marginal perturbations, this construction yields families of defects between a given CFT and all its deformations.

One dimensional objects in two dimensional CFTs like defect lines or boundary conditions can however attract or repel each other. More precisely, there exists a Casimir energy and with it a force between pairs of those objects (see e.g. [3]). This applies in particular to the defects associated to deformations. Hence, extending deformations towards defect lines or boundaries can cost or yield energy. The amount of it depends on the defect or boundary conditions under consideration, but also on the deformation. Thus, any defect line or boundary condition in a given CFT defines a direction in (or rather a tangent vector to) the deformation space of this CFT, by requiring that the energy gain is biggest in this direction. If the defect or boundary 
condition behaves smoothly under all deformations, such that it can be carried to any point in the moduli space, then these vectors form a vector field and hence give rise to a flow on this moduli space. To put it another way, in this case the Casimir energy defines a potential on the moduli space which gives rise to a gradient flow.

Indeed, the gradient vector field can be calculated by means of perturbation theory and can be expressed purely in terms of the coupling of the deforming fields to the chosen defect or boundary condition. Moreover, it turns out, that it is proportional to the gradient vector field of the logarithm of the entropy $g$-function of the defect or boundary condition. That means that the corresponding flow is in fact a constant reparametrization of the gradient flow of $\ln (g)$, and hence drives the bulk moduli of the CFT to local minima or saddle points of the entropy $g$.

An interesting type of fixed points exists for flows associated to defects. Namely, there are special so called 'topological' defect lines ${ }^{1}$, which have the property that correlation functions do not change when the position of these defects are changed as long as no defects or field insertions are crossed. This of course implies that there is no force between them and any other defect or boundary condition. Hence, points in the bulk moduli space, where a defect becomes topological are fixed points of the flow associated to this defect. Moreover, it can be shown that they are in fact attractive fixed points, i.e. minima of the $g$-function.

A related observation was already made in [6] in the context of the free boson CFT. There it was noted that symmetry preserving defects in this model attract each other if and only if fusion decreases their $g$-value. Thus, these defects tend to fuse to ones with lower $g$. In that sense, defects minimizing $g$, which for the free boson are exactly the topological defects are the most stable.

In fact, the flows derived here purely in terms of conformal field theory (at least the ones associated to supersymmetric boundary conditions), also arise in string theory, however from seemingly very different considerations. In the string theory terminology, the parameters of the closed string background evolve under the flow as to decrease the mass of chosen D-branes. Such flows are known as 'attractor flows' in the context of BPS black holes in $N=2$ supergravity [7, 8, 9, 10, 11]. It was noticed that the fixed points of these flows, the 'attractors' correspond to arithmetically interesting geometries and at least in examples to rational world sheet CFTs [11].

As alluded to above, from the world sheet point of view, at least some attractor points of flows associated to defects are points in bulk moduli space, where the defects become topological. For instance, monodromies around Gepner points are described by topological defects (implementing the quantum symmetry) at these points of enhanced symmetry [2]. Away from the Gepner points these defects become nontopological. Therefore, Gepner points are attractors of the flows associated to the monodromy defects.

\footnotetext{
${ }^{1}$ first discussed in the context of RCFT in [4], see [5] for a recent discussion
} 
Another example are $N=(2,2)$ nonlinear sigma models whose target spaces exhibit 'complex multiplication' (see e.g. [12]). Complex multiplications also have a realization as topological defects in the world sheet CFTs. (In specific examples this can be worked out explicitly [13].) Deforming the complex structure of the target spaces away from complex multiplication points, these defects become nontopological. Hence, complex multiplication points are attractive fixed points of the flows on the complex structure moduli spaces, which are associated to the defects. Indeed, classes of manifolds with complex multiplication have been identified as attractors in the supergravity context [11], and complex multiplication has even been proposed as a criterion for rationality of the associated world sheet CFTs $[11,14]$.

It appears that world sheet CFTs associated to general attractor points have special properties, and it would be very interesting to get a better understanding of their nature. The conformal field theory realization of attractor flows considered in this article could shed some light on this question.

Besides, it provides a natural generalization of attractor flows to non-supersymmetric situations which could be of interest in string theory as well.

Flows similar to the ones constructed here using defects also appear in another string theory context, namely in the treatment of backreaction of D-branes on the closed string moduli by means of the Fischler-Susskind mechanism [15, 16]. There, divergences coming from higher genus string amplitudes are compensated by shifts in the coupling constants, which contribute to the RG flow of the bulk world sheet theory. In [17] this was explicitly analyzed for the divergences coming from annulus amplitudes. It is very interesting that the resulting shifted bulk RG flow is indeed closely related to the flows considered here.

The plan of the paper is as follows. In Section 2, a general derivation of the flows is given in terms of Casimir energies. It is shown that they coincide (up to a constant factor) with the gradient flows of the $g$-functions. In Section 3 the example of the free boson compactified on a circle is analyzed in detail. For this theory, the deformation defects can be constructed exactly. The exact expressions for their Casimir energies are compared with the perturbative data needed in the formulation of the flows equations, and flows associated to boundary conditions and defects are discussed. Finally, in Section 4 we comment on the case of $N=(2,2)$ superconformal field theories and compare to the attractor flows arising in the context of black holes in supergravity.

\section{Deformation defects and flows on bulk moduli spaces}

As has been argued in $[1,2]$ to each bulk-perturbation of a conformal field theory, one can associate a unique conformal defect line between the IR and the UV fixed points of the corresponding renormalization group flow. This is obtained by starting with the UV conformal field theory on a surface $\Sigma$, on which one places the identity 
defect Id (i.e. the trivial defect) along a curve $C$ which cuts the surface into two domains $\mathcal{U}_{1}$ and $\mathcal{U}_{2}$. Then one perturbs the conformal field theory, but only on one of these domains $\mathcal{U}_{1}$

$$
\langle\cdots\rangle_{\lambda}=\left\langle\cdots e^{\Delta S}\right\rangle, \quad \Delta S=\sum_{i} \lambda^{i} \int_{\mathcal{U}_{1}} \frac{\mathrm{d}^{2} z}{-2 i} \varphi_{i}(z, \bar{z}) .
$$

The end point of the corresponding renormalization group flow is given by the IR CFT on the domain $\mathcal{U}_{1}$ separated by a non-trivial conformal defect from the UV CFT on $\mathcal{U}_{2}$. Thus, given any bulk flow between two CFTs one obtains in this way a conformal defect line between the IR and UV CFT (c.f. figure 1). Since the original identity defect is transparent in particular to the perturbing fields, no additional regularization is needed on this defect. Therefore, for a given bulk flow this defect is uniquely defined.

Such flow defects in particular exist for exactly marginal perturbations, giving rise to families $\mathcal{D}_{\lambda}$ of conformal defects between a given CFT and all its deformations parametrized by $\lambda$.

Now, one dimensional objects in CFTs such

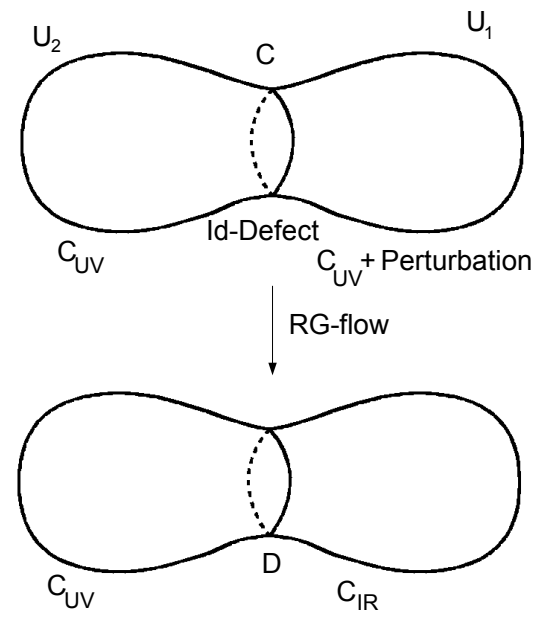

Figure 1: Perturbation on bounded domain gives rise to defect between IRand UV-CFT. as defect lines or boundary conditions repel or attract each other. More precisely, there is a Casimir energy associated to any pair of such objects [3]. This energy can be expressed by means of cylinder amplitudes. Let us first discuss the Casimir energy between a defect line $\mathcal{D}$ and a boundary condition $B$. The amplitude on a half

a)

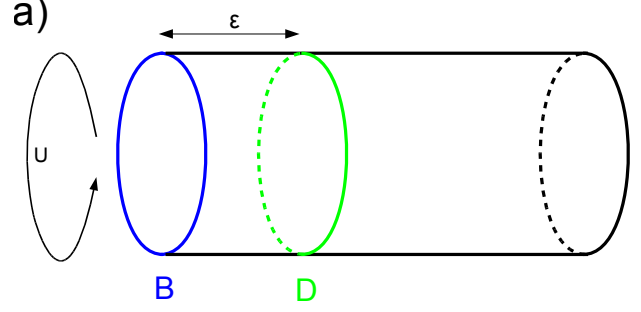

b)

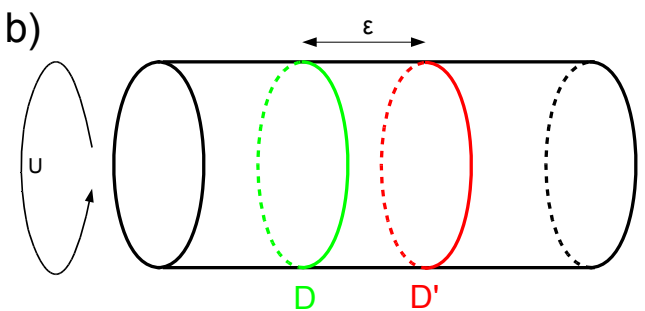

Figure 2: Cylinder amplitudes: a) $\mathcal{B}_{B, \mathcal{D}}^{\epsilon, U}$, and b) $\mathcal{B}_{\mathcal{D}, \mathcal{D}^{\prime}}^{\epsilon, U}$

infinite cylinder with boundary condition $B$ imposed on the finite boundary, defect line $\mathcal{D}$ placed at distance $\epsilon$ parallel to the boundary, and the vacuum $\Omega$ inserted at 
the infinite end of the cylinder (c.f. figure 2a) can be regarded in two dual ways, namely as vacuum transmitted through the defect and absorbed on the boundary, or as loop of half open defect twisted states:

$$
\mathcal{B}_{B, \mathcal{D}}^{\epsilon, U}=\left\langle\left\langle B \| e^{-2 \pi \frac{\epsilon}{U} H} \mathcal{D} \mid \Omega\right\rangle=\operatorname{tr}_{\mathcal{H}_{B, \mathcal{D}}}\left(e^{-2 \pi \frac{U}{\epsilon} H_{B, \mathcal{D}}}\right) .\right.
$$

Here $U$ is the circumference of the cylinder, $2 \pi H$ the bulk Hamiltonian of the CFT, $\mathcal{H}_{B, \mathcal{D}}$ is the Hilbert space of half open twisted states, and $2 \pi H_{B, \mathcal{D}}$ is the Hamiltonian on this space. The ground state energy on $\mathcal{H}_{B, \mathcal{D}}$ can then be obtained as the limit

$$
\mathcal{E}_{B, \mathcal{D}}^{\epsilon}=\frac{\mathcal{E}_{B, \mathcal{D}}}{\epsilon}=-\lim _{U \rightarrow \infty} \frac{1}{U} \ln \left(\mathcal{B}_{B, \mathcal{D}}^{\epsilon, U}\right)
$$

where $\mathcal{E}_{B, \mathcal{D}}$ is the smallest eigenvalue of $2 \pi H_{B, \mathcal{D}}$. This Casimir energy gives rise to a force

$$
\mathcal{F}_{B, \mathcal{D}}^{\epsilon}=-\frac{\partial}{\partial \epsilon} \mathcal{E}_{B, \mathcal{D}}^{\epsilon}=\frac{\mathcal{E}_{B, \mathcal{D}}}{\epsilon^{2}}
$$

between defect and boundary.

If the defect $\mathcal{D}$ is topological, then it commutes with the Hamiltonian, and in particular $\mathcal{B}_{B, \mathcal{D}}$ is independent of $U$. Therefore, the Casimir energy is zero, and there is no force between defect and boundary. General conformal defects however do not commute with $H$ and are attracted or repelled by boundaries. This is true in particular for the defects associated to deformations of CFTs. Hence, any boundary condition in a given CFT defines a direction in the deformation space of this CFT simply by the condition that the deformation defect associated to this direction is the deformation defect attracted the most by the boundary condition. This direction is given by the gradient

$$
-\left.\nabla_{\lambda}\right|_{\lambda=0} \mathcal{E}_{B, \mathcal{D}_{\lambda}}
$$

where $\lambda^{i}$ are local coordinates on the moduli space.

If the boundary condition $B$ is smoothly deformed to $B(\lambda)$ along the bulk deformations $^{2}$, then it defines in this way a vector field and with it a flow on the moduli space of the bulk CFT. Denoting by $\mathcal{D}_{\lambda, \widetilde{\lambda}}$ the deformation defect from theory $\tilde{\lambda}$ to theory $\lambda$ this flow can be written as

$$
\frac{\mathrm{d}}{\mathrm{d} t} \lambda^{i}=-\left.g^{i j} \frac{\partial}{\partial \widetilde{\lambda^{j}}}\right|_{\widetilde{\lambda}=\lambda} \mathcal{E}_{B(\lambda), \mathcal{D}_{\lambda, \tilde{\lambda}}} .
$$

Here $g^{i j}$ is the inverse of the Zamolodchikov metric $g_{i j}=\left\langle\varphi_{i} \mid \varphi_{j}\right\rangle$ on the moduli space.

For deformation defects the gradient (2.4) can of course be calculated by means of perturbation theory

$$
\left.\partial_{\lambda^{j}}\right|_{\lambda=0} \mathcal{E}_{B, \mathcal{D}_{\lambda}}=-\left.\lim _{U \rightarrow \infty} \frac{\epsilon}{U} \frac{\partial_{\lambda^{j}} \mathcal{B}_{B, \mathcal{D}_{\lambda}}^{\epsilon, U}}{\mathcal{B}_{B, \mathcal{D}_{\lambda}}}\right|_{\lambda=0},
$$

\footnotetext{
${ }^{2}$ This is the case if the bulk deformation does not trigger a relevant flow in the boundary sectors, i.e. the bulk-boundary OPE of the deforming bulk fields $\varphi_{j}$ does not give rise to relevant boundary fields.
} 
where

$$
\left.\partial_{\lambda^{j}}\right|_{\lambda=0} \mathcal{B}_{B, \mathcal{D}_{\lambda}}^{\epsilon, U}=\int_{C_{\epsilon, U}} \frac{\mathrm{d}^{2} z}{-2 i}\left\langle\left\langle B \| \varphi_{j}(z, \bar{z}) \mid \Omega\right\rangle,\right.
$$

is the first order term in the perturbative expansion of the amplitude $\mathcal{B}_{B \text {,Id }}^{\epsilon, U}$ perturbed by the field $\varphi_{j}$ on the side of the identity defect which is opposite to the boundary. That means, $C_{\epsilon, U}$ is the half infinite cylinder of circumference $U$ excluding the strip of width $\epsilon$ between the defect and the boundary. This can be more conveniently
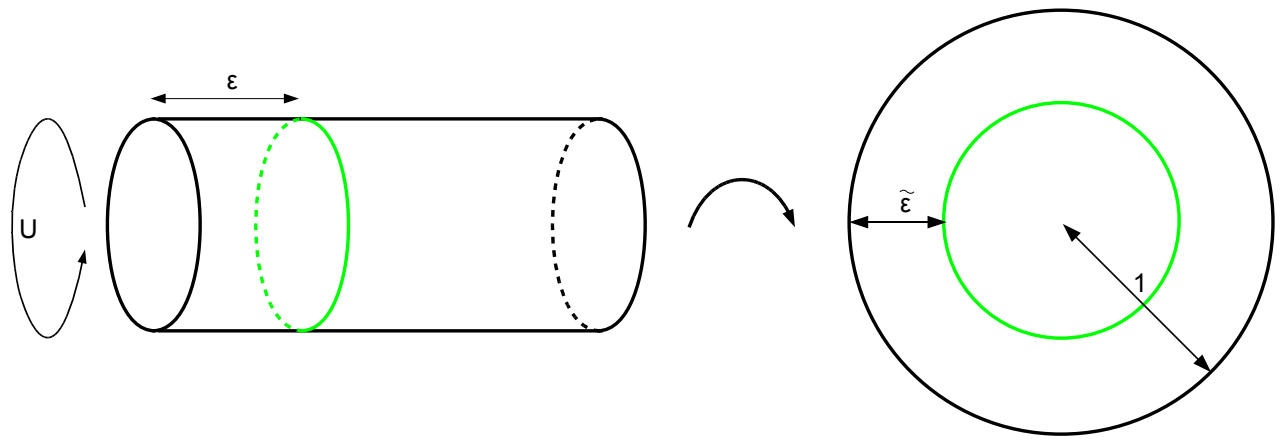

Figure 3: Mapping the cylinder to the unit disk.

calculated on the disk, so we map the half infinite cylinder to the unit disk $D=D_{1}$ by $z \mapsto e^{\frac{2 \pi z}{U}}$ (c.f. figure 3), under which

$$
C_{\epsilon, U} \mapsto D_{1-\tilde{\epsilon}}, \quad 1-\widetilde{\epsilon}=e^{-2 \pi \frac{\epsilon}{U}} .
$$

Since the $\varphi_{j}$ are marginal, i.e. have conformal weights $h_{j}=\bar{h}_{j}=1$, the correlation function on the disk is given by

$$
\left\langle\left\langle B \| \varphi_{j}(z, \bar{z}) \mid \Omega\right\rangle=\frac{g_{B} B_{\varphi_{j}}^{(B)}}{\left(1-|z|^{2}\right)^{2}},\right.
$$

where $g_{B}=\left\langle\langle B \| \Omega\rangle\right.$ is the $g$-factor of the boundary condition $B$ and $B_{\varphi_{j}}^{(B)}:=B_{\varphi_{j}}^{(B) 1}$ is the bulk-boundary OPE coefficient of the perturbing bulk field $\varphi_{j}$ and the identiy $1_{B}$ on the boundary $B$. The integral of this correlation function can be easily computed

$$
\begin{aligned}
\int_{D_{1-\widetilde{\epsilon}}} \frac{\mathrm{d}^{2} z}{-2 i} \frac{1}{\left(1-|z|^{2}\right)^{2}} & =\pi \int_{0}^{(1-\widetilde{\epsilon})^{2}} \mathrm{~d} x(1-x)^{-2}=\pi \frac{(1-\widetilde{\epsilon})^{2}}{1-(1-\widetilde{\epsilon})^{2}} \\
& =\pi \frac{e^{-4 \pi \frac{\epsilon}{U}}}{1-e^{-4 \pi \frac{\epsilon}{U}}} .
\end{aligned}
$$

Since $\mathcal{D}_{0}=\mathrm{Id}$ is the identity defect, and

$$
\lim _{U \rightarrow \infty} \frac{\epsilon}{U} \pi \frac{e^{-4 \pi \frac{\epsilon}{U}}}{1-e^{-4 \pi \frac{\epsilon}{U}}}=\frac{1}{4},
$$


one finds for the gradient

$$
\left.\partial_{\lambda_{j}}\right|_{\lambda=0} \mathcal{E}_{B, \mathcal{D}_{\lambda}}=-\frac{B_{\varphi_{j}}^{(B)}}{4} .
$$

Thus, the flow on bulk moduli space associated to the boundary condition $B$ can be expressed as

$$
\frac{\mathrm{d}}{\mathrm{d} t} \lambda^{i}=\frac{1}{4} g^{i j} B_{\varphi_{j}}^{(B)} .
$$

The fixed points of this flow are those deformations of the bulk theory, in which the perturbing fields do not couple to the chosen boundary condition $B$.

Interestingly, up to a factor $\frac{1}{2 \pi}$, i.e. a constant reparametrization this is nothing but the gradient flow of the logarithm $\ln \left(g_{B}\right)$ of the $g$-function of $B$. This can be seen by means of first order perturbation theory as follows

$$
\left.\partial_{\lambda^{j}}\right|_{\lambda=0} \ln \left(g_{B}\right)=\frac{1}{g_{B}} \int_{D_{1-\widetilde{\epsilon}}} \frac{\mathrm{d}^{2} z}{-2 i}\left\langle\left\langle B \| \varphi_{j}(z, \bar{z}) \mid 0\right\rangle,\right.
$$

where again we have mapped the perturbation from the semi-infinite cylinder to the unit disk. Note however, that here the parameter $\epsilon$ serves as regularization parameter. The relevant one point function on the disk has been given in (2.9) above, and its integral has been calculated in (2.10). Expanding in $\frac{\epsilon}{U}$, one obtains

$$
\int_{D_{1-\tilde{\epsilon}}} \frac{\mathrm{d}^{2} z}{-2 i} \frac{1}{\left(1-|z|^{2}\right)^{2}}=\frac{U}{4 \epsilon}-\frac{\pi}{2}+O\left(\frac{\epsilon}{U}\right) .
$$

In the minimal subraction scheme, renormalization of fields and coupling constants exactly cancels singular terms proportional to $\ln (\epsilon)$ and $\epsilon^{-n}, n>0$. Hence, the remaining finite part is

$$
\left.\partial_{\lambda^{j}}\right|_{\lambda=0} \ln \left(g_{B}\right)=-\frac{\pi}{2} B_{\varphi_{j}}^{(B)},
$$

and the gradient flow of $\ln \left(g_{B}\right)$ is given by

$$
\frac{\mathrm{d}}{\mathrm{d} t} \lambda^{i}=-g^{i j} \partial_{\lambda^{j}} \ln \left(g_{B}\right)=\frac{\pi}{2} g^{i j} B_{\varphi_{j}}^{(B)} .
$$

Comparing with (2.12) we see that the flow obtained by means of the Casimir energy of deformation defects is a constant reparametrization of the gradient flow of $\ln \left(g_{B}\right)$, and hence the flow associated to a boundary condition $B$ decreases its $g$-factor.

As alluded to above, defects are not only attracted or repelled by boundaries but also by other defect lines. Hence, in the same way as boundary conditions also defect lines give rise to flows on bulk moduli spaces. The Casimir energy between two defect lines can be obtained analogously to the boundary case as a limit of a cylinder correlation function $\mathcal{B}_{\mathcal{D}, \mathcal{D}^{\prime}}^{\epsilon,}$. In this case however, the cylinder extends to infinity in both directions with the vacuum inserted at both ends. The two defect lines are placed parallel at distance $\epsilon$ around it (c.f. figure 2b). Analogously to the 
boundary case, this correlation function can be interpreted in two different ways, as the transmission of the vacuum through the two defects or a trace over a Hilbert space $\mathcal{H}_{\mathcal{D}, \mathcal{D}^{\prime}}$ of defect twisted states

$$
\mathcal{B}_{\mathcal{D}, \mathcal{D}^{\prime}}^{\epsilon, U}=\left\langle\Omega\left|\mathcal{D} e^{-2 \pi \frac{\epsilon}{U} H} \mathcal{D}^{\prime}\right| \Omega\right\rangle=\operatorname{tr}_{\mathcal{H}_{\mathcal{D}, \mathcal{D}^{\prime}}}\left(e^{-2 \pi \frac{U}{\epsilon} H_{\mathcal{D}, \mathcal{D}^{\prime}}}\right)
$$

giving rise to the Casimir energy

$$
\mathcal{E}_{\mathcal{D}, \mathcal{D}^{\prime}}^{\epsilon}=\frac{\mathcal{E}_{\mathcal{D}, \mathcal{D}^{\prime}}}{\epsilon}=-\lim _{U \rightarrow \infty} \frac{1}{U} \ln \left(\mathcal{B}_{\mathcal{D}, \mathcal{D}^{\prime}}^{\epsilon, U}\right)
$$

With the same reasoning as for boundary conditions, the Casimir energy between any defect $\mathcal{D}$ and a deformation defect $\mathcal{D}^{\prime}=\mathcal{D}_{\lambda}$ defines a direction in the deformation space of the conformal field theory on one side of the defect line $\mathcal{D}$

$$
-\left.\nabla_{\lambda}\right|_{\lambda=0} \mathcal{E}_{\mathcal{D}, \mathcal{D}_{\lambda}}
$$

Hence, if $\mathcal{D}$ behaves smoothly under the corresponding deformations ${ }^{3}$ it gives rise to a vector field and hence flow on the moduli space of the bulk CFT on one of its sides

$$
\frac{\mathrm{d}}{\mathrm{d} t} \lambda^{i}=-\left.g^{i j} \frac{\partial}{\partial \widetilde{\lambda}^{j}}\right|_{\widetilde{\lambda}=\lambda} \mathcal{E}_{\mathcal{D}(\lambda), \mathcal{D}_{\lambda, \widetilde{\lambda}}}
$$

By means of perturbation theory, this flow can be rewritten as

$$
\frac{\mathrm{d}}{\mathrm{d} t} \lambda^{i}=\frac{1}{4} g^{i j} B_{\varphi_{j}}^{(\mathcal{D})}
$$

where $B_{\varphi_{j}}^{(\mathcal{D})}$ is the OPE coefficient of the perturbing field with the identity on the defect $\mathcal{D}$. This is completely analogous to the boundary case. In fact, the perturbative calculation in the defect case can be reduced to that for boundaries by means of the folding trick ${ }^{4}$. This implies that the flow (2.22) is indeed also a constant reparametrization of the gradient flow

$$
\frac{\mathrm{d}}{\mathrm{d} t} \lambda^{i}=-g^{i j} \partial_{\lambda^{j}} \ln \left(g_{\mathcal{D}}\right)=\frac{\pi}{2} g^{i j} B_{\varphi_{j}}^{(\mathcal{D})} .
$$

of the logarithm of the defect $g$-function defined by

$$
g_{\mathcal{D}}=\langle\Omega|\mathcal{D}| \Omega\rangle .
$$

\footnotetext{
${ }^{3}$ As for boundary conditions this is the case if the bulk deformation does not trigger a relevant flow in the defect sectors, i.e. the bulk-defect OPE of the deforming bulk fields $\varphi_{j}$ does not produce relevant defect fields.

${ }^{4}$ The folding trick maps a correlation function on a cylinder with a defect inserted in the middle to the correlation function on the half cylinder, where one of the halves is folded over to the other one with a respective boundary condition imposed on the boundary thus created $[18,19,3]$. Therefore, the perturbative analysis including bulk-one-point functions is completely analogous.
} 
Note that points $\lambda$, in which $\mathcal{D}$ is a topological defect are fixed points of this flow. This follows from the fact that the Casimir energy between a topological defect and any other defect is zero. In the perturbative approach, bulk-one-point functions in the presence of a topological defect vanish due to conformal covariance, so that $B_{\varphi_{j}}^{(\mathcal{D})}=0$.

It can be shown that points where $\mathcal{D}$ is a topological defect are in fact attractive fixed points, i.e. local minima of $g_{\mathcal{D}}$. As is calculated in Appendix B, the Hessian of $\ln \left(g_{\mathcal{D}}\right)$ at such a point is given by

$$
\left.\partial_{\lambda^{i}} \partial_{\lambda^{j}}\right|_{\lambda=0} \ln \left(g_{\mathcal{D}}\right)=\frac{\pi^{2}}{4} g_{i j}
$$

which is positive definite. Note however that the defect $\mathcal{D}$ is not necessarily topological in all fixed points of the flow Extreme examples of non-topological fixed points are purely reflective defects. These do not transmit any excitations and hence consist of boundary conditions for the theories on each side. In particular they are not topological. The flows associated to such defects are given by the flows associated to the respective boundary conditions, and the fixed points are again purely reflective defects.

Of course, one can also consider deformations on both sides of a defect line $\mathcal{D}$. The relevant Casimir energy can then be expressed in terms of a cylinder amplitude with three defects parallel to each other, the two deformation defects, and $\mathcal{D}$ in between (c.f. figure 4). The corresponding coupled gradient flow is given by

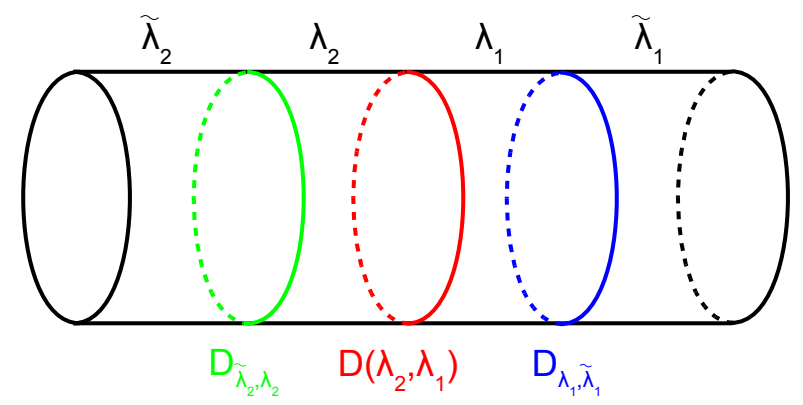

Figure 4: Cylinder with defect $\mathcal{D}$ in between two deformation defects.

$$
\begin{aligned}
\frac{\mathrm{d}}{\mathrm{d} t} \lambda_{1}^{i} & =-\left.g_{1}^{i j} \frac{\partial}{\partial \widetilde{\lambda_{1}^{j}}}\right|_{\widetilde{\lambda}_{i}=\lambda_{i}} \mathcal{E}_{\mathcal{D}_{\widetilde{\lambda}_{2}, \lambda_{2}}, \mathcal{D}\left(\lambda_{2}, \lambda_{1}\right), \mathcal{D}_{\lambda_{1}, \widetilde{\lambda}_{1}}}, \\
\frac{\mathrm{d}}{\mathrm{d} t} \lambda_{2}^{i} & =-\left.g_{2}^{i j} \frac{\partial}{\partial \widetilde{\lambda}_{2}^{j}}\right|_{\widetilde{\lambda}_{i}=\lambda_{i}} \mathcal{E}_{\mathcal{D}_{\widetilde{\lambda}_{2}, \lambda_{2}}, \mathcal{D}\left(\lambda_{2}, \lambda_{1}\right), \mathcal{D}_{\lambda_{1}, \widetilde{\lambda}_{1}}},
\end{aligned}
$$

where $\lambda_{1}^{i}, \widetilde{\lambda}_{1}^{i}$ are the coupling constants on one side of the defect, and $\lambda_{2}^{i}, \widetilde{\lambda}_{2}^{i}$ the ones on the other. First order deformation theory yields

$$
\frac{\mathrm{d}}{\mathrm{d} t} \lambda_{1}^{i}=\frac{1}{4} g_{1}^{i j} B_{\varphi_{j}^{1}}^{(\mathcal{D})}, \quad \frac{\mathrm{d}}{\mathrm{d} t} \lambda_{2}^{i}=\frac{1}{4} g_{2}^{i j} B_{\varphi_{j}^{2}}^{(\mathcal{D})} .
$$

Again, this is a constant reparametrization of the gradient flow of $\ln \left(g_{\mathcal{D}}\right)$. 
If $\mathcal{D}$ is a defect between one and the same theory, then it makes sense to consider simultaneous deformations on both sides, so as to keep it a defect between one and the same theory. The gradient flow is then given by

$$
\frac{\mathrm{d}}{\mathrm{d} t} \lambda^{i}=-\left.g^{i j} \frac{\partial}{\partial \widetilde{\lambda^{j}}}\right|_{\widetilde{\lambda}=\lambda} \mathcal{E}_{\mathcal{D}_{\tilde{\lambda}, \lambda}, \mathcal{D}(\lambda), \mathcal{D}_{\lambda, \tilde{\lambda}}}
$$

In first order one picks up perturbations on both sides of $\mathcal{D}$ and one obtains

$$
\frac{\mathrm{d}}{\mathrm{d} t} \lambda^{i}=\frac{1}{4} g^{i j}\left(B_{\varphi_{j}^{1}}^{(\mathcal{D})}+B_{\varphi_{j}^{2}}^{(\mathcal{D})}\right),
$$

where $\varphi_{j}^{1}$ and $\varphi_{j}^{2}$ refer to the perturbing fields on the two sides of the defect line. By the same arguments as before this is a constant reparametrization of the gradient flow of $\ln \left(g_{\mathcal{D}}\right)$, where here the bulk moduli are varied on both sides of the defect simultaneously.

\section{Example: The free boson}

As an example we consider the free boson compactified on a circle. This conformal field theory is governed by a holomorphic and an antiholomorphic $\widehat{\mathfrak{u}}(1)$ current algebra which are generated by currents

$$
j(z)=\sum_{n} a_{n} z^{-n-1}, \quad \bar{\jmath}(\bar{z})=\sum_{n} \bar{a}_{n} \bar{z}^{-n-1} .
$$

In terms of the currents, the energy momentum tensor can be expressed as

$$
T=\frac{1}{2}: j j:, \quad \bar{T}=\frac{1}{2}: \overline{\jmath \jmath}: .
$$

The Hilbert space of the theory decomposes into the respective highest weight modules $\mathcal{V}_{Q} \otimes \overline{\mathcal{V}}_{\bar{Q}}$ of $\mathfrak{u}(1) \oplus \overline{\mathfrak{u}(1)}$-charge $(Q, \bar{Q})$. The corresponding highest weight vectors $|(Q, \bar{Q})\rangle$ have conformal weights $(h, \bar{h})=\frac{1}{2}\left(Q^{2}, \bar{Q}^{2}\right)$. The model is completely determined by the lattice $\Gamma$ of $\mathfrak{u}(1) \oplus \overline{\mathfrak{u}(1)}$ charges appearing in the theory

$$
\mathcal{H}_{\Gamma}=\bigoplus_{(Q, \bar{Q}) \in \Gamma} \mathcal{V}_{Q} \otimes \overline{\mathcal{V}}_{\bar{Q}}
$$

$\Gamma$ is an even selfdual lattice in $\mathbb{R}^{1,1}$ and it comes in a family

$$
\widetilde{\mathcal{M}}=\mathrm{O}(1,1) / \mathrm{O}(1) \times \mathrm{O}(1) \cong \mathbb{R}^{+}
$$

The latter can be parametrized as

$$
\Gamma_{R}=\left\{\frac{1}{\sqrt{2}}\left(\frac{n}{R}+m R, \frac{n}{R}-m R\right) \mid n, m \in \mathbb{Z}\right\} .
$$


Here $R \in \mathbb{R}^{+}$can be regarded as the radius of the target space circle. The lattices $\Gamma_{R}$ for different $R$ are mapped to each other by means of $\mathrm{O}(1,1)$-transformations, explicitly one has

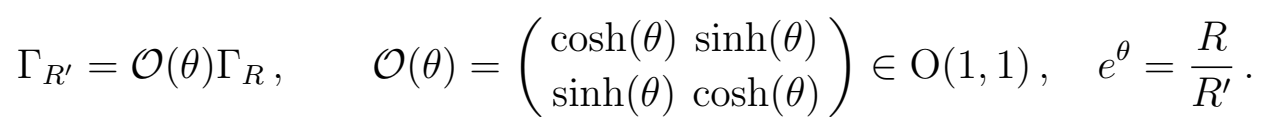

Thus, there is a one-parameter family of free bosonic conformal field theories parametrized by the radius of the target space circle. Indeed, this family can be generated by exactly marginal perturbations with the operator $\varphi=j \bar{\jmath}$ which preserve the $\widehat{\mathfrak{u}}(1) \oplus \overline{\widehat{\mathfrak{u}}}(1)$-current algebra (see e.g. [20]). Perturbing a theory with circle radius $R_{0}$ by adding

$$
\Delta S=\lambda \int_{\Sigma} \mathrm{d}^{2} z j(z) \bar{\jmath}(\bar{z})
$$

to the action one obtains the circle theory with radius

$$
R(\lambda)=R(0) e^{\pi \lambda}
$$

This can be seen by comparing the variation of $\mathfrak{u}(1) \oplus \overline{\mathfrak{u}}(1)$-charges under the perturbation with the $R$-dependence of the charges

$$
\left(Q_{n, m}^{R}, \bar{Q}_{n, m}^{R}\right)=\frac{1}{\sqrt{2}}\left(\frac{n}{R}+m R, \frac{n}{R}-m R\right) .
$$

Namely, by perturbation analysis one finds (see Appendix A)

$$
\partial_{\lambda} Q(\lambda)=-\pi \bar{Q}(\lambda), \quad \partial_{\lambda} \bar{Q}(\lambda)=-\pi Q(\lambda)
$$

whereas

$$
\partial_{\ln (R)} Q_{n, m}^{R}=-\bar{Q}_{n, m}^{R}, \quad \partial_{\ln (R)} \bar{Q}_{n, m}^{R}=-Q_{n, m}^{R}
$$

and hence

$$
\frac{\partial \ln (R)}{\partial \lambda}=\pi
$$

\section{Symmetry-preserving defects}

In this Section we describe the $\widehat{\mathfrak{u}}(1) \oplus \overline{\widehat{\mathfrak{u}}}(1)$-preserving defects between possibly different CFTs in the free boson family (c.f. [3]). The corresponding defect operators $\mathcal{D}: \mathcal{H}_{\Gamma_{1}} \longrightarrow \mathcal{H}_{\Gamma_{2}}$ satisfy gluing conditions

$$
\left(\begin{array}{c}
a_{n}^{2} \\
\bar{a}_{-n}^{2}
\end{array}\right) \mathcal{D}=\mathcal{D} \mathcal{O}\left(\begin{array}{c}
a_{n}^{1} \\
\bar{a}_{-n}^{1}
\end{array}\right)
$$

for $\mathcal{O} \in \mathrm{O}(1,1)$. Here the $a_{n}^{i}$ and $\bar{a}_{n}^{i}$ denote the modes of holomorphic and antiholomorphic $\widehat{\mathfrak{u}}(1)$-currents in theory $i$. If the gluing condition $\mathcal{O}$ is diagonal, then it glues 
together holomorphic and antiholomorphic currents separately, and the resulting defect is topological.

A gluing condition $\mathcal{O}$ is admissible if the lattice

$$
\Gamma_{\mathcal{O}}:=\left\{\gamma \in \Gamma_{1} \mid \mathcal{O} \gamma \in \Gamma_{2}\right\} \subset \Gamma_{1}
$$

of intertwiners $P_{\gamma}^{\mathcal{O}}: \mathcal{V}_{\gamma} \subset \mathcal{H}_{1} \longrightarrow \mathcal{V}_{\mathcal{O}} \subset \mathcal{H}_{2}$ implementing the gluing conditions (3.13) has maximal rank. In this case there are enough intertwiners to construct defect operators

$$
\mathcal{D}_{\mathcal{O}}(\chi):=g_{\mathcal{O}} \sum_{\gamma \in \Gamma_{\mathcal{O}}} P_{\gamma}^{\mathcal{O}} e^{i \chi(\gamma)}
$$

where $\chi \in \Gamma\left(\Gamma_{\mathcal{O}} \otimes \mathbb{R}\right)^{*}$ and

$$
g_{\mathcal{O}}=\sqrt{\left\|\pi_{\mathcal{O}}\left(\Gamma_{\mathcal{O}}\right)\right\|}
$$

is the square root of the volume of the lattice

$$
\pi_{\mathcal{O}}\left(\Gamma_{\mathcal{O}}\right)=\left\{\left(\begin{array}{c}
\pi(\gamma) \\
\bar{\pi}(\mathcal{O} \gamma)
\end{array}\right) \mid \gamma \in \Gamma_{\mathcal{O}}\right\}
$$

Here $\pi$ and $\bar{\pi}$ denote the projections on holomorphic and antiholomorphic charges respectively.

In order to describe the intertwiners in more detail, we make use of the folding trick, which relates defect operators $\mathcal{D}: \mathcal{H}_{1} \longrightarrow \mathcal{H}_{2}$ and boundary states $\left.\left.\| B\right\rangle\right\rangle \in$ $\mathcal{H}_{1}^{*} \otimes \mathcal{H}_{2}$ in the folded theory (see e.g. $[18,19,3]$ ). The latter satisfy gluing conditions

$$
\left.\left.\left(\left(\begin{array}{c}
\widehat{a}_{n}^{1} \\
a_{n}^{2}
\end{array}\right)-S\left(\begin{array}{c}
\widehat{a}_{-n}^{1} \\
\bar{a}_{-n}^{2}
\end{array}\right)\right) \| B\right\rangle\right\rangle=0,
$$

where $S \in \mathrm{O}(2)$ and $\widehat{a}_{n}^{1}$ and $\widehat{\bar{a}}_{n}^{1}$ are the modes of currents of the folded theory which in terms of the ones in the original theory $\operatorname{read}^{5}$

$$
\widehat{a}_{n}^{1}=\bar{a}_{-n}^{1}, \quad \widehat{\bar{a}}_{n}^{1}=a_{-n}^{1} .
$$

Therefore, the gluing conditions in the unfolded and folded theory are related by

$$
S=\frac{1}{\mathcal{O}_{22}}\left(\begin{array}{cc}
-\mathcal{O}_{21} & 1 \\
\operatorname{det}(\mathcal{O}) & \mathcal{O}_{12}
\end{array}\right)
$$

Ishibashi states implementing the gluing condition (3.18) can be written as

$$
e^{\sum_{n>0} \frac{1}{n}\left(S_{11} \widehat{a}_{-n}^{1} \widehat{\bar{a}}_{-n}^{1}+S_{12} \widehat{a}_{-n}^{1} \bar{a}_{-n}^{2}+S_{21} a_{-n}^{2} \widehat{\bar{a}}_{-n}^{1}+S_{22} a_{-n}^{2} \bar{a}_{-n}^{2}\right)}\left|\left(Q_{1}, Q_{2}, \bar{Q}_{1}, \bar{Q}_{2}\right)\right\rangle
$$

\footnotetext{
${ }^{5}$ For convenience of later notation, the currents built out of the $\widehat{a}_{n}^{1}$ and $\widehat{\bar{a}}_{n}^{1}$ are minus the folded currents $j$ and $\bar{\jmath}$ respectively.
} 
for charges satsifying

$$
\left(\begin{array}{l}
Q_{1} \\
Q_{2}
\end{array}\right)=S\left(\bar{Q}_{1} \bar{Q}_{2}\right)
$$

Unfolding yields the intertwiners

$$
P_{x}^{\mathcal{O}}=e^{\sum_{n>0} \frac{1}{n}\left(S_{11} a_{n}^{1} \bar{a}_{n}^{1}+S_{12} a_{n}^{1} \bar{a}_{-n}^{2}+S_{21} a_{-n}^{2} \bar{a}_{n}^{1}+S_{22} a_{-n}^{2} \bar{a}_{-n}^{2}\right)},
$$

where monomials in operators $A_{i}$ of theory $i$ have to be regarded as

$$
A_{1} A_{2}=A_{2} A_{1}=A_{2}|\mathcal{O} x\rangle\langle x| A_{1} .
$$

For $\mathcal{O}=\mathcal{O}(\theta)($ c.f. $(3.6))$

$$
S=\left(\begin{array}{cc}
-\tanh (\theta) & \frac{1}{\cosh (\theta)} \\
\frac{1}{\cosh (\theta)} & \tanh (\theta)
\end{array}\right)
$$

Furthermore,

$$
\pi_{\mathcal{O}}\left(\Gamma_{1}\right)=\left\{\frac{1}{\sqrt{2}}\left(\begin{array}{c}
\frac{n}{R_{1}}+m R_{1} \\
\frac{n}{R_{1}} e^{\theta}-m R_{1} e^{-\theta}
\end{array}\right) \mid n, m \in \mathbb{Z}\right\}
$$

and

$$
\left\|\pi_{\mathcal{O}}\left(\Gamma_{1}\right)\right\|^{2}=\left|\operatorname{det}\left(\begin{array}{cc}
\frac{1}{2 R_{1}^{2}}\left(1+e^{2 \theta}\right) & 0 \\
0 & \frac{R_{1}^{2}}{2}\left(1+e^{-2 \theta}\right)
\end{array}\right)\right|=\cosh ^{2}(\theta) .
$$

Hence

$$
g_{\mathcal{O}}=\sqrt{\cosh (\theta) \operatorname{ind}_{\Gamma_{\mathcal{O}} \subset \Gamma_{1}}} .
$$

The boundary states (3.18) are of course just boundary states on a two torus $T^{2}$ with torus cycles of radius $R_{1}$ and $R_{2}$ respectively. So they correspond either to D1- or D0/D2-branes on this torus. D1-branes are geometrically characterized by winding numbers $\left(k_{1}, k_{2}\right)$ around the two torus cycles; by T-duality they are mapped to bound states of D0- and D2 branes.

The relation between the data used in this paper and the winding numbers is obtained by identifying

$$
e^{-\theta}=\tan \vartheta
$$

where $\vartheta$ is the angle between the D1-brane and the torus cycle of radius $R_{1}$. In terms of $\vartheta$, the gluing condition $S$ of the boundary condition reads ${ }^{6}$

$$
S=\left(\begin{array}{cc}
-\cos 2 \vartheta & \sin 2 \vartheta \\
\sin 2 \vartheta & \cos 2 \vartheta
\end{array}\right)
$$

\footnotetext{
${ }^{6}$ Note that the signs for the off-diagonal matrix entries can be changed by twisting the folding convention (3.19) by $\mathbb{Z}_{2}$ automorphisms of the $\widehat{\mathfrak{u}}(1)$ current algebra.
} 
and for the $g$-factor one obtains

$$
g_{\mathcal{O}}=\sqrt{\frac{k_{1} k_{2}}{\sin 2 \vartheta}}=\sqrt{\frac{k_{1}^{2} R_{1}^{2}+k_{2}^{2} R_{2}^{2}}{2 R_{1} R_{2}}} .
$$

In the last step we have used that

$$
\tan \vartheta=\frac{k_{2} R_{2}}{k_{1} R_{1}}
$$

As it should, the $g$-factor is proportional to the length of the D1-brane.

\section{Deformation defects}

In this Section we identify the defects associated to the exactly marginal bulk perturbation along the family of circle theories. Since this perturbation preserves the holomorphic and anitholomorphic current algebras, also the associated defects should preserve it. Thus, they are among the class of defects discussed in the previous Section. Indeed, as will be argued below, the deformation from a theory with circle radius $R_{2}$ to one with $R_{1}$ is given by the $\widehat{\mathfrak{u}}(1) \oplus \overline{\mathfrak{u}}(1)$-preserving defect $\mathcal{D}_{\mathcal{O}}=\mathcal{D}_{\mathcal{O}}(\chi=0)$ with

$$
\mathcal{O}=\mathcal{O}(\theta), \quad e^{\theta}=\frac{R_{1}}{R_{2}}
$$

In particular,

$$
\Gamma_{\mathcal{O}}=\Gamma_{1}, \quad g_{\mathcal{O}}=\sqrt{\cosh (\theta)}, \quad S=\left(\begin{array}{cc}
-\tanh (\theta) & \frac{1}{\cosh (\theta)} \\
\frac{1}{\cosh (\theta)} & \tanh (\theta)
\end{array}\right) .
$$

In terms of boundary conditions in the folded theory, they correspond to D1-branes stretching diagonally across the two-torus with cycles of radii $R_{1}$ and $R_{2}$.

To see that these are the deformation defects, one has to show that perturbing the theory with radius $R_{2}$ on the half cylinder going to $-\infty$ gives rise to cylinder amplitude with defect $\mathcal{D}_{\mathcal{O}}$ separating the theory with radius $R_{1}$ on the half cylinder extending to $-\infty$ from the original theory with radius $R_{2}$ on the half cylinder extending to $+\infty$. Since we have to compare two families, it is sufficient to do the comparison to first order in all points in the family, i.e. one has to show that $\pi$ times the derivative of amplitudes involving the defect $\mathcal{D}_{\mathcal{O}}$ with respect to $\ln \left(R_{1}\right)$ is equal to the first order deformation with $\varphi=j \bar{\jmath}$ on the $R_{1}$-side of the defect

$$
\pi \partial_{\ln \left(R_{1}\right)}\left\langle\cdots \mathcal{D}_{\mathcal{O}} \cdots\right\rangle=\int_{C} \frac{\mathrm{d}^{2} z}{-2 i}\left\langle\cdots \mathcal{D}_{\mathcal{O}} j(z) \bar{\jmath}(\bar{z}) \cdots\right\rangle .
$$

Since we know that the deformation defect preserves the current algebra, it is sufficient to show this for the amplitudes defining the action of the defect operator on highest weight states and the ones encoding the gluing conditions. 
For the former consider the cylinder amplitude

$$
\mathcal{A}_{R_{1}, R_{2}}^{\gamma_{1}, \gamma_{2}}={ }_{R_{2}}\left\langle\gamma_{2}\left|\mathcal{D}_{\mathcal{O}}\right| \gamma_{1}\right\rangle_{R_{1}}=g_{\mathcal{O}} \delta_{\gamma_{2}, \mathcal{O} \gamma_{1}}
$$

Deriving with respect to $\ln \left(R_{1}\right)$ one obtains ${ }^{7}$

$$
\partial_{\ln \left(R_{1}\right)} \mathcal{A}_{R_{1}, R_{2}}^{\gamma_{1}, \gamma_{2}}=\partial_{\theta} \mathcal{A}_{R_{1}, R_{2}}^{\gamma_{1}, \gamma_{2}}=\frac{1}{2} \tanh (\theta) \mathcal{A}_{R_{1}, R_{2}}^{\gamma_{1}, \gamma_{2}}
$$

On the other hand, the first order perturbation calculated on the complex plane with the defect placed on the unit circle is given by

$$
\left.\partial_{\lambda}\right|_{\lambda=0} \mathcal{A}_{R_{1}, R_{2}}^{\gamma_{1}, \gamma_{2}}(\lambda)=\int_{D_{1}} \frac{\mathrm{d}^{2} z}{-2 i} R_{2}\left\langle\gamma_{2}\left|\mathcal{D}_{\mathcal{O}} j(z) \bar{\jmath}(\bar{z})\right| \gamma_{1}\right\rangle_{R_{1}} .
$$

Now, using the form (3.23) of the intertwiners one easily obtains

$$
\begin{aligned}
R_{2}\left\langle\gamma_{2}\left|\mathcal{D}_{\mathcal{O}} j(z) \bar{\jmath}(\bar{z})\right| \gamma_{1}\right\rangle_{R_{1}} & ={ }_{R_{1}}\left\langle\mathcal{O}^{-1} \gamma_{2}\left|e^{\sum_{n>0} \frac{S_{11}}{n} a_{n}^{1} \bar{a}_{n}^{1}} \sum_{r, s} a_{r} z^{-r-1} \bar{a}_{s} \bar{z}^{-s-1}\right| \gamma_{1}\right\rangle_{R_{1}} \\
& =\left(\frac{Q \bar{Q}}{|z|^{2}}+\frac{S_{11}}{\left(1-|z|^{2}\right)^{2}}\right) \mathcal{A}_{R_{1}, R_{2}}^{\gamma_{1}, \gamma_{2}}
\end{aligned}
$$

Introducing the spacial cutoff $\widetilde{\epsilon}$, the first summand gives a contribution

$$
I_{1}=\int_{D_{1}-D_{\widetilde{\epsilon}}} \frac{\mathrm{d}^{2} z}{-2 i|z|^{2}}=-2 \pi \ln (\widetilde{\epsilon}),
$$

whereas the second summand yields

$$
I_{2}=\int_{D_{1-\widetilde{\epsilon}}} \frac{\mathrm{d}^{2} z}{-2 i} \frac{1}{\left(1-|z|^{2}\right)^{2}}=\pi \frac{(1-\widetilde{\epsilon})^{2}}{1-(1-\widetilde{\epsilon})^{2}} .
$$

Transforming back to the cylinder $z \mapsto w=\ln (z)$, the cutoff $\tilde{\epsilon}$ on the disk can be expressed in the cutoff $\epsilon$ on the cylinder

$$
1-\widetilde{\epsilon}=e^{-2 \pi \epsilon}
$$

Thus, the contributions to the cylinder are

$$
I_{1}=-2 \pi \ln \left(1-e^{-2 \pi \epsilon}\right)=O(\epsilon), \quad I_{2}=\pi \frac{e^{-4 \pi \epsilon}}{1-e^{-4 \pi \epsilon}}=\frac{1}{4 \epsilon}-\frac{\pi}{2}+O(\epsilon) .
$$

We use the minimal subtraction scheme, in which renormalization of fields and coupling constants are defined in such a way that they exactly cancel the singularities $\epsilon^{-n}, n>0$ and $\ln (\epsilon)$. Thus, we obtain

$$
\left.\partial_{\lambda}\right|_{\lambda=0} \mathcal{A}_{R_{1}, R_{2}}^{\gamma_{1}, \gamma_{2}}(\lambda)=-\frac{\pi}{2} S_{11} \mathcal{A}_{R_{1}, R_{2}}^{\gamma_{1}, \gamma_{2}}=\frac{\pi}{2} \tanh (\theta) \mathcal{A}_{R_{1}, R_{2}}^{\gamma_{1}, \gamma_{2}}
$$

\footnotetext{
${ }^{7}$ For $\gamma_{1}$ we take a constant section in the family, i.e. the argument of the $\delta$-function is constant.
} 
Comparing with (3.37) we indeed find that equation (3.35) is satisfied for the amplitudes $\mathcal{A}_{R_{1}, R_{2}}^{\gamma_{1}, \gamma_{2}}$ defining the action of the defect operator on highest weight states.

As a next step we show that the same is true for amplitudes encoding the gluing conditions. The latter can be probed by cylinder amplitudes of descendents of the vacuum. We will present the calculation for

$$
G_{R_{1}, R_{2}}={ }_{R_{2}}\left\langle 0\left|a_{1}^{2} \mathcal{D}_{\mathcal{O}} a_{-1}^{1}\right| 0\right\rangle_{R_{1}}=\frac{\operatorname{det}(\mathcal{O})}{\mathcal{O}_{22}} R_{2}\left\langle 0\left|\mathcal{D}_{\mathcal{O}}\right| 0\right\rangle_{R_{1}}=\frac{1}{\sqrt{\cosh (\theta)}}
$$

The calculation for the remaining gluing amplitudes can be performed in a similar fashion. The derivative of this amplitude with respect to $\ln \left(R_{1}\right)$ is given by

$$
\partial_{\ln \left(R_{1}\right)} G_{R_{1}, R_{2}}=\partial_{\theta} G_{R_{1}, R_{2}}=-\frac{1}{2} \frac{\sinh (\theta)}{\cosh ^{2}(\theta)} g_{\mathcal{O}}
$$

Again, first order perturbation theory on the disk yields

$$
\left.\partial_{\lambda}\right|_{\lambda=0} G_{R_{1}, R_{2}}=\int_{D_{1}} \frac{\mathrm{d}^{2} z}{-2 i} R_{2}\left\langle 0\left|a_{1}^{2} \mathcal{D}_{\mathcal{O}} j(z) \bar{\jmath}(\bar{z}) a_{-1}^{1}\right| 0\right\rangle_{R_{1}} .
$$

The correlation function can be easily calculated to be

$$
R_{2}\left\langle 0\left|a_{1}^{2} \mathcal{D}_{\mathcal{O}} j(z) \bar{\jmath}(\bar{z}) a_{-1}^{1}\right| 0\right\rangle_{R_{1}}=-\frac{\sinh (\theta)}{\cosh ^{2}(\theta)} g_{\mathcal{O}}\left(1+\frac{1}{\left(1-|z|^{2}\right)^{2}}\right)
$$

The integral $I_{2}$ over the second summand has already been calculated (c.f. (3.43)), and the integral over the first summand 1 of course yields $\pi$. Thus, one obtains

$$
\left.\partial_{\lambda}\right|_{\lambda=0} G_{R_{1}, R_{2}}=-\frac{\pi}{2} \frac{\sinh (\theta)}{\cosh ^{2}(\theta)}
$$

which agrees with $\pi$ times the derivative (3.46). Hence, the defects $\mathcal{D}_{\mathcal{O}}$ are indeed the deformation defects along the family of the free bosonic CFTs.

\section{Casimir energy from boundary conditions}

Next, we calculate explicitly the Casimir energy between deformation defects $\mathcal{D}_{\mathcal{O}}$ and $\mathfrak{u}(1)$-preserving boundary conditions. The corresponding boundary states obey gluing conditions

$$
\left.\left.\left(a_{n}-T \bar{a}_{-n}\right) \| B\right\rangle\right\rangle^{T}=0, \quad T \in \mathrm{O}(1) \cong \mathbb{Z}_{2}
$$

and are given by

$$
\| B(\chi)\rangle\rangle^{T}=g^{T} \sum_{\gamma \in \Gamma^{T}} e^{i \chi(\gamma)} e^{\sum_{n>0} \frac{T}{n} a_{-n} \bar{a}_{-n}}|\gamma\rangle
$$


The gluing condition $T$ is +1 for Dirichlet and -1 for Neumann boundary conditions respectively. Moreover,

$$
\Gamma^{T}=\Gamma \cap\{(T x, x) \mid x \in \mathbb{R}\}
$$

denotes the lattice of Ishibashi states,

$$
g^{T}=\sqrt{\left\|\pi\left(\Gamma^{T}\right)\right\|}
$$

is the square root of the volume of the projection of $\Gamma^{T}$ on the holomorphic charges, and $\chi \in\left(\Gamma^{T} \otimes \mathbb{R}\right)^{*}$ determines position or Wilson line in case of Dirichlet and Neumann boundary conditions, respectively. For a free boson on a circle of radius $R$, one obtains

$$
\Gamma^{T}=\frac{1}{\sqrt{2} R^{T}} \mathbb{Z}, \quad g^{T}=\frac{1}{\sqrt{\sqrt{2} R^{T}}} .
$$

The Casimir energy between a defect $\mathcal{D}$ and a boundary condition $\| B\rangle\rangle$ can be obtained from the amplitude on a semi-infinite cylinder of circumference $U$ with the boundary condition imposed on the finite end of the cylinder, the defect placed a distance $\epsilon$ away from it and the vacuum inserted at the infinite end of the cylinder (c.f. figure $2 \mathrm{a}$ )

$$
\mathcal{B}_{B, \mathcal{D}}^{\epsilon, U}=\left\langle\left\langle B \| e^{-2 \pi \frac{\epsilon}{U}\left(L_{0}+\bar{L}_{0}\right)} \mathcal{D} \mid \Omega\right\rangle\right.
$$

Namely,

$$
\mathcal{E}_{B, \mathcal{D}}^{\epsilon}=-\lim _{U \rightarrow \infty} \frac{1}{U} \ln \left(\mathcal{B}_{B, \mathcal{D}}^{\epsilon, U}\right)
$$

For $\mathfrak{u}(1)$-preserving defects and boundary conditions in the free boson theory, this can be easily calculated (c.f. $[3,6])$. The cylinder amplitude is given by

$$
\begin{aligned}
\mathcal{B}_{B^{T}, \mathcal{D}_{\mathcal{O}}}^{\epsilon, U} & =g_{\mathcal{O}} g^{T} R_{2}\langle 0| e^{\sum_{n>0} \frac{T}{n} a_{n}^{2} \bar{a}_{n}^{2}} e^{-2 \pi \frac{\epsilon}{U}\left(L_{0}+\bar{L}_{0}\right)} e^{\sum_{n>0} \frac{S_{22}}{n} a_{-n}^{2} \bar{a}_{-n}^{2}|0\rangle_{R_{2}}} \\
& =g_{\mathcal{O}} g^{T} \prod_{n>0} R_{2}\langle 0| e^{\frac{T}{n} a_{n}^{2} \bar{a}_{n}^{2}} e^{-2 \pi \frac{\epsilon}{U}\left(L_{0}+\bar{L}_{0}\right)} e^{\frac{S_{22} a_{-n}^{2} \bar{a}_{-n}^{2}}{n}|0\rangle_{R_{2}}} \\
& =g_{\mathcal{O}} g^{T} \prod_{n>0} \sum_{l \geq 0} \frac{1}{(l !)^{2}}\left(\frac{T S_{22} e^{-4 \pi n \frac{\epsilon}{U}}}{n^{2}}\right)^{l} R_{2}\left\langle 0\left|\left(a_{n}^{2} \bar{a}_{n}^{2}\right)^{l}\left(a_{-n}^{2} \bar{a}_{-n}^{2}\right)^{l}\right| 0\right\rangle_{R_{2}} \\
& =g_{\mathcal{O}} g^{T} \prod_{n>0} \sum_{l \geq 0}\left(T S_{22} e^{-4 \pi n \frac{\epsilon}{U}}\right)^{l} \\
& =g_{\mathcal{O}} g^{T} \prod_{n>0} \frac{1}{1-T S_{22} e^{-4 \pi n \frac{\epsilon}{U}}} .
\end{aligned}
$$

Using the Euler-MacLaurin formula, one finds the exact expression of the Casimir energy

$$
\mathcal{E}_{B^{T}, \mathcal{D}_{\mathcal{O}}}^{\epsilon}=\frac{\mathrm{Li}_{2}\left(T S_{22}\right)}{4 \pi \epsilon}, \quad \mathcal{E}_{B^{T}, \mathcal{D}_{\mathcal{O}}}=\epsilon \mathcal{E}_{B^{T}, \mathcal{D}_{\mathcal{O}}}^{\epsilon}=\frac{\mathrm{Li}_{2}\left(T S_{22}\right)}{4 \pi}
$$

in terms of the dilogarithm $[3,6]$. 


\section{Flows induced by boundary conditions}

Since the boundary conditions $B^{T}$ preserve the $\widehat{\mathfrak{u}}(1)$ current algebras, they transform along smoothly under deformations generated by ${ }^{8} \varphi=j \bar{\jmath}$, and in particular their gluing conditions $T$ do not change.

Thus, a boundary condition $B^{T}$ gives rise to a flow on the moduli space of the free boson CFT as described in Section 2. From the exact formula (3.58) it is easy to obtain ${ }^{9}$

$$
\left.\partial_{\ln \left(R_{1}\right)}\right|_{R_{1}=R_{2}} \mathcal{E}_{B^{T}, \mathcal{D}_{\mathcal{O}}}=\left.\partial_{\theta}\right|_{\theta=0} \mathcal{E}_{B^{T}, \mathcal{D}_{\mathcal{O}}}=-\frac{T}{4 \pi} .
$$

This agrees with the general form (2.12) of the first order perturbative calculation of the derivative of $\mathcal{E}$, where $B_{j \bar{\jmath}}^{\left(B^{T}\right)}=T$ and $\partial_{\lambda}=\pi \partial_{\ln (R)}$.

In fact, one does not need the dilogarithm formula (3.58) to arrive at this result. By means of

$$
\begin{aligned}
\left.\partial_{\ln \left(R_{1}\right)}\right|_{R_{1}=R_{2}} \mathcal{E}_{B^{T}, \mathcal{D}_{\mathcal{O}}} & =\left.\partial_{\theta}\right|_{\theta=0} \mathcal{E}_{B^{T}, \mathcal{D}_{\mathcal{O}}}=-\left.\lim _{U \rightarrow \infty} \frac{\epsilon}{U} \frac{\partial_{\theta} \mathcal{B}_{B^{T}, \mathcal{D}_{\mathcal{O}}}^{\epsilon, U}}{\mathcal{B}_{B^{T}, \mathcal{D}_{\mathcal{O}}}^{\epsilon,}}\right|_{\theta=0} \\
& =-\left.\lim _{U \rightarrow \infty} \frac{\epsilon}{U g^{T}} \partial_{\theta} \mathcal{B}_{B^{T}, \mathcal{D}_{\mathcal{O}}}^{\epsilon, U}\right|_{\theta=0}
\end{aligned}
$$

it can be directly calculated from formula (3.57)

$$
\left.\partial_{\theta} \mathcal{B}_{B^{T}, \mathcal{D}_{\mathcal{O}}}^{\epsilon,}\right|_{\theta=0}=g^{T} g_{\mathcal{O}} T \frac{e^{-4 \pi \frac{\epsilon}{U}}}{1-e^{-4 \pi \frac{\epsilon}{U}}},
$$

yielding the same result

$$
\left.\partial_{\ln \left(R_{1}\right)}\right|_{R_{1}=R_{2}} \mathcal{E}_{B^{T}, \mathcal{D}_{\mathcal{O}}}=-\frac{T}{4 \pi} .
$$

The corresponding flow is then given by

$$
\frac{\mathrm{d}}{\mathrm{d} t} \lambda=\frac{T}{4},
$$

with solutions

$$
\lambda(t)=\frac{T t}{4}, \quad R(t)=R(0) e^{\frac{\pi T t}{4}} .
$$

That means that for Dirichlet boundary conditions $(T=1)$ the radius flows to $\infty$, whereas for Neumann boundary conditions $(T=-1)$ the radius flows to 0 . This is expected, after all we have argued in Section 2 that these flows are in general constant reparametrizations of gradient flows for $\ln \left(g^{T}\right)$, hence decrease $g^{T}$. Thus, Dirichlet boundary conditions drive the target space circle radius to $\infty$, whereas Neumann boundary conditions drive it to 0 .

\footnotetext{
${ }^{8}$ This is due to the fact that the perturbing field $\varphi=j \bar{\jmath}$ via bulk-boundary OPE does not induce relevant perturbations on the boundary.

${ }^{9} \partial_{x} \mathrm{Li}_{2}(x)=\frac{\ln (1-x)}{x}$
} 


\section{Casimir energy from defects}

The Casimir energy between two defects $\mathcal{D}$ and $\mathcal{D}^{\prime}$ can be obtained from the amplitude

$$
\mathcal{B}_{\mathcal{D}, \mathcal{D}^{\prime}}^{\epsilon, U}=\left\langle\Omega\left|\mathcal{D} e^{-2 \pi \frac{\epsilon}{U}\left(L_{0}+\bar{L}_{0}\right)} \mathcal{D}^{\prime}\right| \Omega\right\rangle
$$

on an infinite cylinder of circumference $U$ with the vacuum inserted at both ends and the two defects placed parallel at distance $\epsilon$ on it (c.f. figure 2b). Namely,

$$
\mathcal{E}_{\mathcal{D}, \mathcal{D}^{\prime}}^{\epsilon}=-\lim _{U \rightarrow \infty} \frac{1}{U} \ln \left(\mathcal{B}_{\mathcal{D}, \mathcal{D}^{\prime}}^{\epsilon, U} .\right.
$$

For two $\mathfrak{u}(1)$-preserving defects $\mathcal{D}=\mathcal{D}_{\mathcal{O}}$ between free boson theories of radii $R_{3}$ and $R_{2}$ and $\mathcal{D}^{\prime}=\mathcal{D}_{\mathcal{O}^{\prime}}$ between theories with radii $R_{2}$ and $R_{1}$ this amplitude can easily be calculated. Indeed, the calculation is analogous to the calculation (3.57) in the boundary case with the result

$$
\begin{aligned}
\mathcal{B}_{\mathcal{D}, \mathcal{D}^{\prime}}^{\epsilon, U} & =g_{\mathcal{O}} g_{\mathcal{O}^{\prime} R_{2}}\left\langle\Omega\left|e^{\sum_{n>0} \frac{S_{11}}{n} a_{n}^{2} \bar{a}_{n}^{2}} e^{-2 \pi \frac{\epsilon}{U}\left(L_{0}+\bar{L}_{0}\right)} e^{\sum_{n>0} \frac{S_{22}^{\prime}}{n} a_{-n}^{2} \bar{a}_{-n}^{2}}\right| 0\right\rangle_{R_{2}} \\
& =g_{\mathcal{O}} g_{\mathcal{O}^{\prime}} \prod_{n>0} \frac{1}{1-S_{11} S_{22}^{\prime} e^{-4 \pi n \frac{\epsilon}{U}}} .
\end{aligned}
$$

As in the boundary case this leads to the Casimir energy

$$
\mathcal{E}_{\mathcal{D}, \mathcal{D}^{\prime}}^{\epsilon}=\frac{\mathrm{Li}_{2}\left(S_{11} S_{22}^{\prime}\right)}{4 \pi \epsilon}, \quad \mathcal{E}_{\mathcal{D}, \mathcal{D}^{\prime}}=\frac{\operatorname{Li}_{2}\left(S_{11} S_{22}^{\prime}\right)}{4 \pi}
$$

Flows defined by defects

Also $\widehat{\mathfrak{u}}(1)$-preserving defects $\mathcal{D}_{\mathcal{O}}$ behave smoothly under deformations generated by ${ }^{10}$ $\varphi=j \bar{\jmath}$. Thus, a defect $\mathcal{D}_{\mathcal{O}}$ gives rise to a flow on the moduli space of the free boson theory on one of its sides. Taking for $\mathcal{D}^{\prime}$ a deformation defect, similarly to the boundary case, one easily obtains the derivatives of the Casimir energy

$$
\left.\partial_{\ln \left(R_{1}\right)}\right|_{R_{1}=R_{2}} \mathcal{E}_{\mathcal{D}, \mathcal{D}^{\prime}}=\left.\partial_{\theta^{\prime}}\right|_{\theta^{\prime}=0} \mathcal{E}_{\mathcal{D}, \mathcal{D}^{\prime}}=-\frac{S_{11}}{4 \pi} .
$$

Again this agrees with the general perturbative formula (2.22), where

$$
B_{\varphi}^{(\mathcal{D})}=S_{11}=-\frac{\mathcal{O}_{21}}{\mathcal{O}_{22}}
$$

In contrast to the boundary case, the gluing condition $\mathcal{O}$ of the defect $\mathcal{D}$ is not constant under the deformation of the theory on one side, but is deformed according to $^{11}$

$$
\mathcal{O} \mapsto \mathcal{O O}(\pi \lambda)
$$

\footnotetext{
${ }^{10}$ The bulk-defect OPE of the perturbing field $\varphi=j \bar{\jmath}$ does not produce relevant defect fields.

${ }^{11}$ This can be seen for instance by fusing the defect with the corresponding deformation defect under which the gluing conditions multiply.
} 
In particular, the relevant coupling constant $S_{11}$ depends on the deformation parameter. Writing the undeformed gluing condition as

$$
\mathcal{O}=A_{s_{1}, s_{2}} \mathcal{O}(\theta)
$$

with $s_{1}, s_{2} \in \mathbb{Z}_{2}$ and

$$
A_{s_{1}, s_{2}}=\left(\begin{array}{cc}
(-1)^{s_{1}} & 0 \\
0 & (-1)^{s_{2}}
\end{array}\right)
$$

the deformed gluing condition is given by

$$
A_{s_{1}, s_{2}} \mathcal{O}(\theta(\lambda))=A_{s_{1}, s_{2}} \mathcal{O}(\theta+\pi \lambda)
$$

Hence,

$$
S_{11}(\lambda)=-\tanh (\theta(\lambda))=-\tanh (\theta(0)+\pi \lambda) .
$$

Therefore, the flow defined by the defect $\mathcal{D}_{\mathcal{O}}$ is given by

$$
\frac{\mathrm{d}}{\mathrm{d} t} \lambda=\frac{S_{11}(\lambda)}{4}=-\frac{1}{4} \tanh (\theta(0)+\pi \lambda)
$$

It can be integrated to

$$
\sinh (\theta(\lambda))=\sinh (\theta(0)+\pi \lambda)=\sinh (\theta(0)) e^{-\pi \frac{t}{4}}
$$

and one immediately finds that that the gluing condition $\mathcal{O}$ flows to the diagonal one in its connected component, which corresponds to a topological defect. This is expected. After all the flow is a reparametrization of the gradient flow of

$$
\ln \left(g_{\mathcal{O}}\right)=\frac{1}{2} \ln \operatorname{ind}_{\Gamma_{\mathcal{O}} \subset \Gamma_{1}}+\frac{1}{2} \ln \cosh (\theta)
$$

and $\cosh (\theta)$ of course only has a single critical point, its minimum in $\theta=0$, where $\sinh (\theta=0)=0$.

An 'attractor mechanism' for defects in the compactified free boson has already been proposed in [6], where the full fusion product between two arbitrary symmetry preserving defects was worked out. It was noted that the difference in $\ln (g)$ before and after fusion, which was interpreted as entropy release has the same sign as the Casimir force between the two defects. In particular, defects are attracted to each other, when $\ln (g)$ decreases under fusion. In this sense, the minima of $\ln (g)$, which for the free boson correspond to topological defects were suggested as attractors.

Let us also briefly discuss the simultaneous flow on both sides of a defect between one and the same theory. For these flows the theories on both sides of the defect remain identical during the flow. The flow equation (2.29) in this case become

$$
\frac{\mathrm{d}}{\mathrm{d} t} \lambda=\frac{1}{4}\left(S_{11}+S_{22}\right)
$$


Here,

$$
B_{\varphi_{1}}^{(\mathcal{D})}=S_{11}=-\frac{\mathcal{O}_{21}}{\mathcal{O}_{22}}=-\tanh (\theta), \quad B_{\varphi_{2}}^{(\mathcal{D})}=S_{22}=\frac{\mathcal{O}_{12}}{\mathcal{O}_{22}}=(-1)^{s_{1}+s_{2}} \tanh (\theta)
$$

But under simultaneous deformations, the gluing condition $\mathcal{O}=A_{s_{1}, s_{2}} \mathcal{O}(\theta)$ of a defect behaves as

$$
\mathcal{O} \mapsto \mathcal{O}(-\pi \lambda) \mathcal{O O}(\pi \lambda)=A_{s_{1}, s_{2}} \mathcal{O}\left(\theta+\pi \lambda\left(1-(-1)^{s_{1}+s_{2}}\right)\right)
$$

Thus, the flow equation becomes

$$
\frac{\mathrm{d}}{\mathrm{d} t} \lambda=-\frac{1}{4}\left(1-(-1)^{s_{1}+s_{2}}\right) \tanh \left(\theta+\pi \lambda\left(1-(-1)^{s_{1}+s_{2}}\right)\right) .
$$

It can be integrated to

$$
\sinh (\theta(\lambda))=\sinh \left(\theta(0)+\pi \lambda\left(1-(-1)^{s_{1}+s_{2}}\right)\right)=\sinh (\theta(0)) e^{-\frac{\pi t}{4}\left(1-(-1)^{s_{1}+s_{2}}\right)^{2}} .
$$

Hence, there is no flow for even $s_{1}+s_{2}$. This is expected, because in this case simultaneous deformation does not change the gluing condition, and therefore $g$ is constant. For odd $s_{1}+s_{2}$ on the other hand, $\lambda$ flows to a point, where the gluing condition becomes diagonal, i.e. the defect becomes topological.

\section{Supersymmetric version and attractor flows}

The construction of flows on CFT moduli spaces outlined in Section 2 of course can be applied to supersymmetric conformal field theories as well. Indeed, supersymmetry preserving deformations are more robust and hence easier to deal with than generic ones. Moreover, $N=(2,2)$ SCFTs and their moduli spaces are of particular interest in string theory.

$N=(2,2)$ superconformal field theories admit two classes of supersymmetry preserving bulk perturbations, those coming from chiral primary fields with the same chirality in the holomorphic and the anti-holomorphic sectors, i.e. (chiral,chiral)and (anti-chiral, anti-chiral)-primary fields, and those coming from chiral primaries with opposite chirality, i.e. (a,c)- and (c,a)-primary fields. For non-linear sigma models these correspond respectively to deformations of the complex structure and the Kähler structure of the target space.

While all these perturbations (they will be referred to respectively as (c,c)- and (a,c)-perturbations in the following) preserve the $N=(2,2)$ supersymmetry of the bulk theory, they generically cease to be supersymmetric in the presence of boundaries or defect lines. 
More precisely, supersymmetric boundary conditions and defects come in two classes as well, A-type and B-type, depending on which of the $N=2$ superconformal subalgebras of the bulk superconformal algebra they preserve ${ }^{12}$. It is well known that (c,c)-perturbations preserve supersymmetry on A-type boundary conditions and defects, while they generically destroy supersymmetry on boundary conditions and defects of B-type. Analogously, (a,c)-perturbations preserve supersymmetry on Btype boundary condtions and defects, but generically do not preserve supersymmetry on boundary conditions and defects of A-type. (See [24, 25] for details on the boundary case and [1] for comments on defects.) This applies in particular to exactly marginal perturbations.

Given a supersymmetric boundary condition or defect in some $N=(2,2)$ superconformal field theory, the construction in Section 2 gives rise to a direction (2.12) in the deformation space of the underlying bulk SCFT. In fact, for A-type boundary conditions or defects this direction is a (c,c)-direction, whereas for B-type boundary conditions or defects it is an (a,c)-direction, and thus, the corresponding deformation preserves supersymmetry. This is due to the fact that the relevant couplings of (a,c)-/(c,c)-perturbing fields to A-/B-type boundary conditions or defects vanish. For boundary conditions this has already been shown in [26], and the defect case can be treated similarly. (The argument is sketched in Appendix C.)

However, while all marginal $(\mathrm{c}, \mathrm{c})$ - or $(\mathrm{a}, \mathrm{c})$-perturbations are exactly marginal in the bulk, they can induce relevant perturbations on boundaries and defects, even in the case supersymmetry is preserved [24, 25]. This happens at 'lines of marginal stability' in moduli space. Away from these lines, the chosen defect or boundary condition behaves smoothly under deformations, and by the construction presented in Section 2 gives rise to a flow on the (c,c)- or (a,c)-moduli space.

As discussed in Section 2, the flows are constant reparametrizations of gradient flows of $\ln (g)$, where the ground state degeneracy $g$ only depends on the topological charges of the corresponding boundary condition or defect. The latter are well defined for any supersymmetric boundary condition or defect irrespective of conformal invariance. Thus, the gradient flows are well defined on the entire moduli space.

In the case of non-linear sigma models with Calabi-Yau target manifold, A-type boundary conditions corresponding to A-branes on Lagrangian submanifolds give rise to flows on the complex structure moduli space of the target space. B-type boundary conditions, which are given by coherent sheaves on the target space on the other hand induce flows on the Kähler moduli space.

Viewed in the context of string compactifications, the resulting theory in four dimensions is an $N=2$ supergravity theory where (for type IIB) the (a,c)-moduli become scalars in the hypermultiplets, and the (c,c)-moduli in the vector multiplets.

\footnotetext{
${ }^{12}$ Defects can preserve the entire $N=(2,2)$-superconformal algebra, i.e. they can be of A-type and of B-type at the same time [21]. B-type defects have recently been investigated in the context of Landau-Ginzburg models, where they are easily constructed explicitely [22, 21, 23]
} 
D-branes can be viewed as black holes of this supergravity theory provided that the boundary conditions are Dirichlet in the uncompactified directions. The black hole solution a priori depends on the background moduli as well as the electric and magnetic charges they carry. The latter are determined by the topological charges of the A-type boundary condition in the internal theory, which can be expressed in terms of the homology class, the corresponding A-brane represents in the compactification space. The values of the moduli at the horizon of the black hole can be obtained as fixed points of a flow on the respective moduli space [7]. This flow is referred to as attractor flow in supergravity. It can be realized as the gradient flow of the logarithm $\ln \left(|Z|^{2}\right)$ of the absolute value squared of the central charge of the corresponding low energy BPS particle (see e.g. [11, 27]).

The mass of a BPS particle in four dimensions equals the absolute value $|Z|$ of the central charge. Since the graviton vertex operator is the identity in the compactified dimensions, the mass of a BPS particle is given by the g-function of the corresponding boundary condition in the internal theory. As a consequence the absolute value of the central charge is equal to the g-function ${ }^{13}$

$$
|Z|=g_{B}
$$

Therefore, the attractor flow is a constant reparametrization of the gradient flow of $\ln \left(g_{B}\right)$. The construction of flows by means of Casimir energies presented in Section 2 therefore provides a world sheet realization of the attractor flow encountered in supergravity. Besides, it also generalizes the attractor flow in various directions. For instance, it associates flows not only to D-branes, but also to defects. Moreover, it does not require supersymmetry, nor any target space interpretation.

\section{Acknowledgements}

D. R. is supported by a DFG research fellowship and partially by DOE-grant DEFG02-96ER40949. I. B. is supported by a EURYI award. We would like to thanks N. Carqueville, A. Collinucci and S. Nampuri for discussions.

\section{A. Perturbative variation of charges}

It is well known that bilinear combinations of $\mathfrak{u}(1)$-currents generate exactly marginal perturbations (see e.g. [20]). To determine the first order variations of $\mathfrak{u}(1)$-charges under such deformations we introduce a space-time cutoff $\epsilon$ and use the minimal subtraction scheme with respect to this cutoff. That means the renormalizations of fields and coupling constants are chosen in such a way that they cancel exactly

\footnotetext{
${ }^{13}$ The phase of the central charge is encoded in the boundary condition of the spectral flow operator, from which one constructs the space-time supercharges.
} 
the singularities proportional to $\ln (\epsilon)$ and $\epsilon^{-n}, n>0$. (For more details on this see e.g. the Appendix of [28].)

The variation of the $\mathfrak{u}(1)$-charges can be determined by calculating the first order perturbation of the correlation function

$$
\langle(Q, \bar{Q})|j(z)|(Q, \bar{Q})\rangle=\frac{Q}{z}\langle(Q, \bar{Q}) \mid(Q, \bar{Q})\rangle=\frac{Q}{z} .
$$

One obtains

$$
\begin{aligned}
\left.\partial_{\lambda}\right|_{\lambda=0}\langle(Q, \bar{Q})|j(z)|(Q, \bar{Q})\rangle & =\frac{1}{-2 i} \int_{\mathbb{P}^{1}-D_{\epsilon}(0)-D_{\epsilon}(z)} \mathrm{d}^{2} w\langle(Q, \bar{Q})|j(w) \bar{\jmath}(\bar{w}) j(z)|(Q, \bar{Q})\rangle \\
& =-\frac{\bar{Q}}{2 i} \int_{\mathbb{P}^{1}-D_{\epsilon}(0)-D_{\epsilon}(z)} \mathrm{d}^{2} w\left(\frac{1}{(z-w)^{2} \bar{w}}+\frac{Q^{2}}{|w|^{2} z}\right),
\end{aligned}
$$

where $D_{\epsilon}(z)$ is the disk with radius $\epsilon$ around $z$. The integral over the second summand gives rise to a term proportional to $\ln (\epsilon)$ which is compensated by a field redefinition of $|(Q, \bar{Q})\rangle$. The first term can be rewritten by means of

$$
\frac{1}{(z-w)^{2} \bar{w}}=\partial_{w} \frac{1}{(z-w) \bar{w}}
$$

which holds outside the singularities. Thus, one obtains

$$
\begin{aligned}
\left.\partial_{\lambda}\right|_{\lambda=0}\langle(Q, \bar{Q})|j(z)|(Q, \bar{Q})\rangle & =-\frac{\bar{Q}}{2 i} \int_{\mathbb{P}^{1}-D_{\epsilon}(0)-D_{\epsilon}(z)} \mathrm{d} \frac{\mathrm{d} \bar{w}}{(z-w) \bar{w}} \\
& =\frac{\bar{Q}}{2 i} \int_{\mathbb{S}_{\epsilon}^{1}(0)+\mathbb{S}_{\epsilon}^{1}(z)} \frac{\mathrm{d} \bar{w}}{(z-w) \bar{w}} \\
& =-\frac{\bar{Q}}{2 i} \int_{\mathbb{S}_{\epsilon}^{1}(0)} \frac{\mathrm{d} w}{w(z-w)}+\frac{\bar{Q}}{2 i} \int_{\mathbb{S}_{\epsilon}^{1}(z)} \frac{\mathrm{d} \bar{w}}{\bar{w}(z-w)} \\
& =-\frac{\pi \bar{Q}}{z}
\end{aligned}
$$

where in the last step, the second summand is zero in the limit $\epsilon \rightarrow 0$. Hence, $\partial_{\lambda} Q=-\pi \bar{Q}$, and in the same way, one arrives at $\partial_{\lambda} \bar{Q}=-\pi Q$.

\section{B. The Hessian of $\ln \left(g_{\mathcal{D}}\right)$ for topological defects}

Since $\ln \left(g_{\mathcal{D}}\right)$ is critical in topological defects, in those points we have

$$
\begin{aligned}
\left.\partial_{\lambda^{i}} \partial_{\lambda^{j}}\right|_{\lambda=0} \ln \left(g_{\mathcal{D}}\right) & =\left.\frac{1}{g_{\mathcal{D}}} \partial_{\lambda^{i}} \partial_{\lambda^{j}}\right|_{\lambda=0} g_{\mathcal{D}} \\
& =\frac{1}{g_{\mathcal{D}}} \int \frac{\mathrm{d}^{2} z}{-2 i} \int \frac{\mathrm{d}^{2} w}{-2 i}\left\langle\varphi_{i}(w, \bar{w}) \varphi_{j}(z, \bar{z})\right\rangle_{\mathcal{D}}
\end{aligned}
$$


where the correlation function is one on the complex plane with defect $\mathcal{D}$ placed on the unit circle, and the integrals are over the unit disk with regularization inherited from the cylinder geometry.

Since the defect is topological, the correlation function is given by

$$
\left\langle\varphi_{i}(w, \bar{w}) \varphi_{j}(z, \bar{z})\right\rangle_{\mathcal{D}}=\frac{g_{\mathcal{D}} g_{i j}}{|z-w|^{4}}
$$

The integral

$$
I:=\int \frac{\mathrm{d}^{2} z}{-2 i} \int \frac{\mathrm{d}^{2} w}{-2 i} \frac{1}{|z-w|^{4}}
$$

can be calculated. Expansion in the regularization parameter $\epsilon$ yields the finite part $I_{\text {reg }}=\frac{\pi^{2}}{4}$. Hence,

$$
\left.\partial_{\lambda^{i}} \partial_{\lambda^{j}}\right|_{\lambda=0} \ln \left(g_{\mathcal{D}}\right)=\frac{\pi^{2}}{4} g_{i j},
$$

which for unitary theories is positive definite.

\section{Vanishing of bulk-boundary/-defect couplings for $N=2$ theories}

Here we briefly sketch why the couplings of (a,c)-perturbing fields to A-type boundaries and defects, and analogously the couplings of (c,c)-perturbing fields to B-type boundaries and defects vanish. The argument for the case of boundary conditions has already been given in [26]. It can be easily adapted to the treatment of defects. Let us discuss the bulk-boundary coupling of an (a,c)-perturbing field $\varphi$ to an A-type boundary vanishes. The other cases can be dealt with analogously.

The perturbing field $\varphi$ is a descendant of an (a,c)-primary $\chi$, i.e.

$$
\varphi=G_{-\frac{1}{2}}^{+} \bar{G}_{-\frac{1}{2}}^{-} \chi, \quad \text { with } \quad G_{-\frac{1}{2}}^{-} \chi=0=\bar{G}_{-\frac{1}{2}}^{+} \chi .
$$

Here $G^{ \pm}$and $\bar{G}^{ \pm}$are the holomorphic and anti-holomorphic supercurrents respectively. Now, consider the one-point function

$$
\langle\varphi(0)\rangle_{A}=g_{A} B_{\varphi}^{(A)}
$$

of $\varphi$ on the unit disk with an A-type boundary condition $A$ imposed on the unit circle. The action of $G^{+}$on $\chi$ can be written as a contour integral

$$
\left(G_{-\frac{1}{2}}^{+} \bar{G}_{-\frac{1}{2}}^{-} \chi\right)(0)=\oint \frac{\mathrm{d} z}{2 \pi i} G^{+}(z)\left(\bar{G}_{-\frac{1}{2}}^{-} \chi\right)(0)
$$

around 0 inside the unit disk. The contour can be deformed to the boundary, where A-type gluing conditions require $G^{+}=\bar{G}^{-}$. Thus,

$$
\begin{aligned}
\langle\varphi(0)\rangle_{A} & =\oint \frac{\mathrm{d} z}{2 \pi i}\left\langle G^{+}(z)\left(\bar{G}_{-\frac{1}{2}}^{-} \chi\right)(0)\right\rangle_{A}=-\oint \frac{\mathrm{d} \bar{z}}{2 \pi i}\left\langle\bar{G}^{-}(\bar{z})\left(\bar{G}_{-\frac{1}{2}}^{-} \chi\right)(0)\right\rangle_{A}(\mathrm{C} .4) \\
& =\left\langle\left(\left(\bar{G}_{-\frac{1}{2}}^{-}\right)^{2} \chi\right)(0)\right\rangle_{A},
\end{aligned}
$$


which vanishes because $\left(\bar{G}_{-\frac{1}{2}}^{-}\right)^{2}=0$. This shows that the coupling $B_{\varphi}^{(A)}$ of an (a,c)-perturbing field $\varphi$ to an A-type boundary condition is zero.

\section{References}

[1] I. Brunner and D. Roggenkamp, "Defects and Bulk Perturbations of Boundary Landau-Ginzburg Orbifolds," JHEP 04 (2008) 001, 0712.0188.

[2] I. Brunner, H. Jockers, and D. Roggenkamp, "Defects and D-Brane Monodromies," 0806.4734.

[3] C. Bachas, J. de Boer, R. Dijkgraaf, and H. Ooguri, "Permeable conformal walls and holography," JHEP 06 (2002) 027, hep-th/0111210.

[4] V. B. Petkova and J. B. Zuber, "Generalised twisted partition functions," Phys. Lett. B504 (2001) 157-164, hep-th/0011021.

[5] J. Fröhlich, J. Fuchs, I. Runkel, and C. Schweigert, "Duality and defects in rational conformal field theory," Nucl. Phys. B763 (2007) 354-430, hep-th/0607247.

[6] C. Bachas and I. Brunner, "Fusion of conformal interfaces," JHEP 02 (2008) 085, arXiv:0712.0076 [hep-th].

[7] S. Ferrara, R. Kallosh, and A. Strominger, "N=2 extremal black holes," Phys. Rev. D52 (1995) 5412-5416, hep-th/9508072.

[8] S. Ferrara and R. Kallosh, "Universality of Supersymmetric Attractors," Phys. Rev. D54 (1996) 1525-1534, hep-th/9603090.

[9] S. Ferrara and R. Kallosh, "Supersymmetry and Attractors," Phys. Rev. D54 (1996) 1514-1524, hep-th/9602136.

[10] S. Ferrara, G. W. Gibbons, and R. Kallosh, "Black holes and critical points in moduli space," Nucl. Phys. B500 (1997) 75-93, hep-th/9702103.

[11] G. W. Moore, "Arithmetic and attractors," hep-th/9807087.

[12] C. Borcea, "Calabi-yau threefolds and complex multiplication," in Essays on Mirror Manifolds, S.-T. Yau, ed. International Press, 1992.

[13] D. Roggenkamp, "Defects, rationality and complex multiplication." unpublished.

[14] S. Gukov and C. Vafa, "Rational conformal field theories and complex multiplication," Commun. Math. Phys. 246 (2004) 181-210, hep-th/0203213.

[15] W. Fischler and L. Susskind, "Dilaton Tadpoles, String Condensates and Scale Invariance. 2," Phys. Lett. B173 (1986) 262. 
[16] W. Fischler and L. Susskind, "Dilaton Tadpoles, String Condensates and Scale Invariance," Phys. Lett. B171 (1986) 383.

[17] C. A. Keller, "Brane backreactions and the Fischler-Susskind mechanism in conformal field theory," JHEP 12 (2007) 046, 0709.1076.

[18] E. Wong and I. Affleck, "Tunneling in quantum wires: A Boundary conformal field theory approach," Nucl. Phys. B417 (1994) 403-438.

[19] M. Oshikawa and I. Affleck, "Defect Lines in the Ising Model and Boundary States on Orbifolds," Phys. Rev. Lett. 77 (1996) 2604-2607, hep-th/9606177.

[20] S. Chaudhuri and J. A. Schwartz, "A criterion for integrably marginal operators," Phys. Lett. B219 (1989) 291.

[21] I. Brunner and D. Roggenkamp, "B-type defects in Landau-Ginzburg models," JHEP 08 (2007) 093, arXiv:0707.0922 [hep-th].

[22] A. Kapustin and L. Rozansky, "On the relation between open and closed topological strings," Commun. Math. Phys. 252 (2004) 393-414, hep-th/0405232.

[23] N. Carqueville and I. Runkel, "On the monoidal structure of matrix bi-factorisations," arXiv:0909.4381.

[24] I. Brunner, M. R. Gaberdiel, S. Hohenegger, and C. A. Keller, "Obstructions and lines of marginal stability from the world-sheet," JHEP 05 (2009) 007, 0902.3177.

[25] M. R. Gaberdiel and S. Hohenegger, "Manifestly Supersymmetric RG Flows," 0910.5122.

[26] H. Ooguri, Y. Oz, and Z. Yin, "D-branes on Calabi-Yau spaces and their mirrors," Nucl. Phys. B477 (1996) 407-430, hep-th/9606112.

[27] F. Denef, "Supergravity flows and D-brane stability," JHEP 08 (2000) 050, hep-th/0005049.

[28] S. Förste and D. Roggenkamp, "Current current deformations of conformal field theories, and WZW models," JHEP 05 (2003) 071, hep-th/0304234. 